\title{
Internal biliary diversion to avoid liver transplantation in an adult with intractable pruritus due to idiopathic intrahepatic cholestasis
}

R. C. Siriwardana*, D. S. P. Jayatunge, C. S. Ekanayake, S. Tilakaratne, M. A. Niriella, B. Gunathilake and A. S. Dassanayake

\begin{abstract}
Background: Cholestasis is due to the obstruction at any level of the excretory pathway of bile. One particularly troublesome symptom of cholestasis is pruritus which leads to a profound effect on a patient's quality of life. In children with progressive familial intrahepatic cholestasis (PFIC), medical treatment often fails. An alternative surgical procedure using biliary diversion offers significant relief for intractable pruritus in non-responders.

Case presentation: A 43-year-old male presented with a history of persistent jaundice and intractable pruritus for a 2-month duration. His liver enzymes were markedly elevated. However, his liver synthetic function was preserved. After extensive evaluation, a cause for cholestasis was not identified. A multi-disciplinary decision was to consider liver transplantation, but as his liver synthetic functions were remarkably preserved and the intractable pruritus was the sole indication for a transplantation, it was finally decided that internal biliary diversion should be done for symptomatic relief. His pruritus dramatically improved at 6 weeks post-operative. His liver enzymes and bilirubin levels also decreased compared to his pre-operative status. Currently, his liver functions are being closely monitored.

Conclusion: Though it is not used in adults, the experience of biliary diversion in children with PFIC shows that there is a considerable improvement of symptoms and postpone the need for a transplant. In our patient, liver function and bilirubin as expected did not show a major improvement. But the dramatic improvement of the symptoms gave us the time to postpone the liver transplantation. A biliary diversion is a reasonable option that needs to be considered even in adults with refectory pruritus due to cholestasis.
\end{abstract}

Keywords: Liver dysfunction, Quality of life, Jaundice

\section{Background}

Bile acids synthesized in hepatocytes or absorbed from the sinusoidal blood are transported and secreted into biliary canaliculi and channeled to the duodenum. These are actively reabsorbed from the terminal ileum and transported into sinusoids completing entero-hepatic

\footnotetext{
*Correspondence: rohansiriwardana@yahoo.com

Department of Surgery, Faculty of Medicine, University of Kelaniya, P.O Box 6, Thalagolla Road, Ragama, Sri Lanka
}

\section{Springer Open}

(c) The Author(s). 2020 Open Access This article is licensed under a Creative Commons Attribution 4.0 International License, which permits use, sharing, adaptation, distribution and reproduction in any medium or format, as long as you give appropriate credit to the original author(s) and the source, provide a link to the Creative Commons licence, and indicate if changes were made. The images or other third party material in this article are included in the article's Creative Commons licence, unless indicated otherwise in a credit line to the material. If material is not included in the article's Creative Commons licence and your intended use is not permitted by statutory regulation or exceeds the permitted use, you will need to obtain permission directly from the copyright holder. To view a copy of this licence, visit http://creativecommons.org/licenses/by/4.0/. ling condition due to an obstruction at any level of the excretory pathway of bile, usually seen in certain instances of viral, alcoholic, drug-induced, chronic liver diseases, and congenital conditions [1]. Despite extensive investigations in certain cases, the cause of cholestasis is not determined. One particularly troublesome symptom associated with cholestasis is pruritus, pruritus can have a profound effect on a patient's quality of life, resulting 
in sleep deprivation and emotional disturbances sometimes ending up in liver transplantation [2].

In children with progressive familial intrahepatic cholestasis (PFIC), an inherited progressive cholestatic disorder characterized by intrahepatic cholestasis and intense pruritus, medical treatment often fails. An alternative surgical procedure using biliary diversion offers significant relief for intractable pruritus in nonresponders [3, 4]. Biliary diversion decreases the pool of bile acids in the enterohepatic circulation by $50 \%$, bypassing the terminal ileal reabsorption and hence decrease the preload to the biliary canaliculi [5]. Currently, there are no published data on using biliary diversion as an alternative for liver transplant in adults to relieve intractable pruritus due to cholestasis.

\section{Case presentation}

A 43-year-old previously healthy male presented with a history of persistent jaundice and intractable pruritus for a 2-month duration. He had no childhood or previous history of similar episodes of symptoms. On examination, he was deeply icteric, pale, and emaciated with a BMI of $17.3 \mathrm{~kg} / \mathrm{m}^{2}$. His liver enzymes (AST $<$ ALT, total bilirubin, alkaline phosphatase) were markedly elevated. However, his liver synthetic function was well preserved with INR 0.92 and albumin of $4.1 \mathrm{~g} / \mathrm{dl}$.

He was extensively evaluated for the cause of cholestasis. He had a normal hepatitis viral serology, serum copper, ceruloplasmin, and anti-mitochondrial antibody levels. On radiological assessment ultrasound scan, contrast-enhanced CT and MRCP showed no evidence of intrahepatic or extrahepatic bile duct dilatation. The liver biopsy showed marked canalicular and intracellular cholestasis with no significant fibrosis and inflammation.
Gradually, the patient's bilirubin level and pruritus became worse. Pruritus was so intense where it was not possible for him to maintain a normal life despite being treated with multiple drug combination with cholestyramine, ursodeoxycholic acid, Naltrexone, and Rifampicin. A trail of a high dose of prednisolone was also given with no improvement in his condition.

His case was discussed in a multi-disciplinary meeting focusing on proceeding for liver transplantation. As his liver synthetic functions were remarkably preserved and the intractable pruritus was the sole indication for a transplantation, it was decided to discuss the possibility of internal biliary diversion. The patient eventually consented to the procedure.

A right-sided upper transverse incision was made. For the cholecysto-jejunocolic anastomosis, a 40-cm midjejunal loop was isolated, and this conduit was taken retrocolic to the supracolic compartment. The proximal end of the conduit was anastomosed to the gall bladder fundus, and the distal end was anastomosed to the proximal transverse colon using end to side anastomosis (Fig. 1). The proximal transverse colon was chosen for the distal anastomosis as it was more technically accessible for a tension-free conduit.

Postoperatively on day 7 , he developed an attack of cholangitis which was treated with antibiotics. His pruritus dramatically improved from a visual analog scale of $10 / 10$ to $1 / 10$ at 6 weeks [6]. His liver enzymes, bilirubin levels, alkaline phosphate, and gamma GT also decreased compared to his pre-operative status. Currently, his liver functions are being closely monitored.

\section{Discussion}

A biliary diversion was first described for intractable pruritus associated with progressive familial intrahepatic

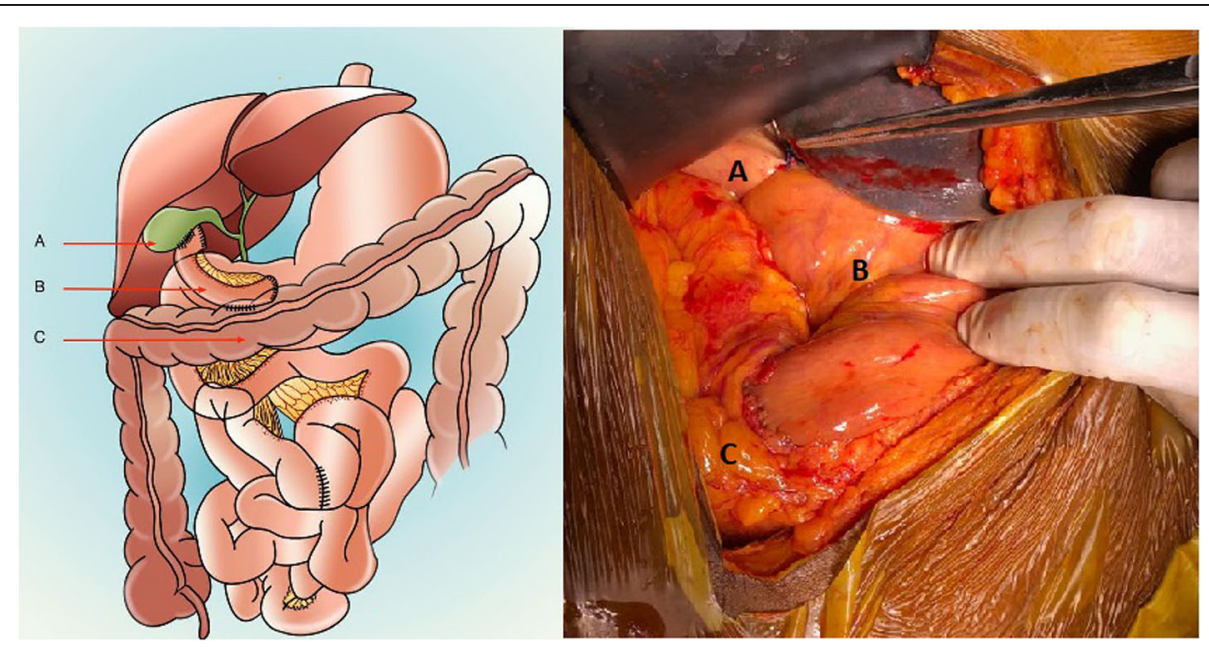

Fig. 1 Biliary diversion: A 40-cm mid jejunal loop (b) was taken retrocolic to the supracolic compartment. Superiorly to the gall bladder fundus (a) and inferiorly to the proximal transverse colon (c) was anastomosed to the conduit 
cholestasis (PFIC) in pediatric patients [7]. Whitington and Whitington in 1988 introduced cholecystojejunocutaneostomy as a form of partial external biliary diversion (PEBD). Holland et al. proposed an ileal bypass to avoid the complications faced by the stoma in PEBD [4, 8]. Bustorff-Silva et al. promoted cholecysto-jejuno-colic anastomosis in two children as a partial internal biliary diversion to avoid a stoma and prevent malabsorption [4]. Though this procedure is not widely practiced, it is well known to improve the intractable pruritus in children with PFIC. It has been shown that there is a significant post-procedure reduction of serum bile salt levels and bilirubin levels. Complete or partial improvement of pruritus is well documented. However, no improvement in disease progression or liver histology is expected after the procedure. It is a procedure that will buy time for transplantation by controlling intractable pruritus.

The exact mechanism of pruritus in cholestasis is not clearly understood, and it is believed to be related to bile salts [9]. However, the degree of symptom is unrelated to the serum bile salt levels indicating that there are other mechanisms involved. The two most common cholestatic liver diseases are PSC and PBC. Pruritus itself is an indication for liver transplantation in them. Dramatic improvement in the quality of life is well documented in them [10]. However, there are other common groups of patients presenting with cholestasis without underlying liver parenchymal injury like in our case [11]. Especially, this group of patients has the likelihood of improvement of symptoms given adequate time. The biliary diversion is a commendable final option in these patients that can be used to buy time.

\section{Conclusion}

Though it is not used in adults, the experience of the biliary diversion in children with PFIC shows that there is considerable improvement of symptoms, postpone the need for a transplant, and in few rare instances completely avoided the transplantation [12]. The improvement of the symptoms is believed to be a result of diminishing the circulating bile salt pool. It is a simple procedure that can be easily reversed. In our patient, liver function and bilirubin as expected did not show a major improvement [6]. But the dramatic improvement of the symptoms gave us the time to postpone the liver transplantation. A biliary diversion is a reasonable option that needs to be considered even in adults with refectory pruritus due to cholestasis.

\section{Abbreviations}

AST: Aspartate transaminase; ALT: Alanine transaminase; CT: Computed tomography; MRCP: Magnetic resonance cholangiopancreatography; PFIC: Progressive familial intrahepatic cholestasis

Acknowledgements

Not applicable

\section{Authors' contributions}

RCS - Drafting the work or revising it critically for important intellectual content and wrote the article. DSPJ - Drafting the work or revising it critically for important intellectual content and wrote the article. CSE - Drafting the work or revising it critically for important intellectual content. ST - Substantial contributions to the conception or design of the work. MAN - Substantial contributions to the conception or design of the work. BG - Drafting the work or revising it critically for important intellectual content. AD -

Substantial contributions to the conception or design of the work. The authors have read and approved the manuscript.

\section{Funding}

No funding

Availability of data and materials

All data generated or analyzed during this study are included in this published article.

Ethics approval and consent to participate

Verbal consent was given by the participant.

\section{Consent for publication}

The patient included in this case report gave written informed consent to publish the data included in this case report.

The patient consent to publish form is available and is submitted as a supplementary report.

\section{Competing interests}

The authors declare that they have no competing interests.

Received: 6 July 2020 Accepted: 24 August 2020

Published online: 10 September 2020

\section{References}

1. Mazokopakis EE, Papadakis JA, Kofteridis DP (2007) Unusual causes of intrahepatic cholestatic liver disease. World J Gastroenterol 13(12):1879-1882

2. Kremer AE, Elferink RPJO, Beuers U (2011) Pathophysiology and current management of pruritus in liver disease. Clinics and Research in Hepatology and Gastroenterology 35:89-97

3. Gunaydin M, Cil ATB (2018) Progressive familial intrahepatic cholestasis: diagnosis, management, and treatment. Hepatic Medicine: Evidence and Research 10:95-104

4. Ganesh R, Suresh N, Sathiyasekeran M et al (2011) Partial internal biliary diversion: a solution for intractable pruritus in progressive familial intrahepatic cholestasis type 1. Saudi I Gastroenterol 17:212-214

5. Melter M, Rodeck B, Kardorff RR et al (2000) Progressive familial intrahepatic cholestasis: partial biliary diversion normalizes serum lipids and improves growth in noncirrhotic patients. Am J Gastroenterol 95:3522-3528

6. Patrick DL, Bush JW, Chen MM (1973) Methods for measuring levels of wellbeing for a health status index. Health Serv Res 8:228-245

7. Whitington PF, Whitington GL (1988 Jul) Partial external diversion of bile for the treatment of intractable pruritus associated with intrahepatic cholestasis. Gastroenterology. 95(1):130-136

8. Silva JB, Neto LS, Olimpio $\mathrm{H}$ et al (2007) Partial internal biliary diversion through a cholecystojejunocolonic anastomosis-a novel surgical approach for patients with progressive familial intrahepatic cholestasis: a preliminary report. J Pediatr Surg 42:1337-1340

9. Geenes V, Williamson C (2009) Intrahepatic cholestasis of pregnancy. World J Gastroenterol 15:2049-2066

10. Gross CR, Malinchoc M, Kim WR et al (1999 Feb) Quality of life before and after liver transplantation for cholestatic liver disease. Hepatology. 29(2):356-364

11. Heathcote EJ (2007 Jul) Diagnosis and management of cholestatic liver disease. Clin Gastroenterol Hepatol 5(7):776-782

12. Gunaydin M, Tander B, Demirel D et al (2016 Mar) Different techniques for biliary diversion in progressive familial intrahepatic cholestasis. J Pediatr Surg 51(3):386-389

\section{Publisher's Note}

Springer Nature remains neutral with regard to jurisdictional claims in published maps and institutional affiliations. 